\title{
NQR Study of Dynamics in Incommensurate Phases
}

\author{
V. S. S. Sastry, K. Venu, S. Uma Maheswari ${ }^{a}$, and R. K. Subramanian ${ }^{\mathrm{b}}$ \\ School of Physics, University of Hyderabad, Hyderabad 500046, India \\ a Mahindra British Telecom Ltd., Pimpri, Pune, India \\ b Department of Physics, University of Utah, Salt Lake City, Utah, USA \\ Reprint requests to Prof. V. S. S. S., Fax: +91-40-3010120; E-mail: vssssp@uohyd.ernet.in
}

Z. Naturforsch. 55a, 281-290 (1999); received September 11, 1999 Presented at the XVth International Symposium on Nuclear Quadrupole Interactions,
Leipzig, Germany, July 25-30, 1999.

Dynamic processes in solids exhibiting structurally incommensurate phases are briefly reviewed, and the application of NMR and NQR is discussed. The unique utility of these methods, - arising due to, on one hand, the microscopic resonant nature of the probe used and, on the other, the presence of periodic, though incommensurable, structure -, is brought out by presenting recent results in a prototype system $\left(\mathrm{Rb}_{2} \mathrm{ZnCl}_{4}\right)$ in the presence of randomly quenched disorder. In particular, the interesting new methodology of measuring, by analysing NQR spin echo modulation, ultra-slow diffusion like collective motions of ensembles of atoms in the presence of pinning effects due to disorder is illustrated with new results.

\section{Introduction}

Incommensurate (IC) phases of insulators arise from competing short range forces and are characterised by the appearance of a long periodic spatial distortion bearing no rational relation to the underlying lattice periodicity. Several compounds of the $\mathrm{A}_{2} \mathrm{BX}_{4}$ family $(\mathrm{A}=\mathrm{K}$, $\mathrm{NH}_{4}, \mathrm{Rb}, \mathrm{Cs} ; \mathrm{B}=\mathrm{Zn}, \mathrm{Cd}, \mathrm{Hg} ; \mathrm{X}=\mathrm{Cl}, \mathrm{Br}, \mathrm{I}$ ) exhibit structural phase transitions (say, at $T_{\mathrm{I}}$ ) to IC phases [1]. The order parameter excitation spectrum of such IC systems consists of an acoustic-like phason branch and an opticlike amplitudon branch [2]. According to the continuum theory in the plane wave limit (PWL) [2], the phason is gapless in the absence of pinning and represents the Goldstone mode recovering the broken translational symmetry (of the free energy) of the IC phase. Development of incommensurate order with decreasing temperature in this phase leads to the breakdown of the plane wave approximation and the realisation of phase solitons (domain walls separating essentially commensurate regions) [3,4]. The discrete nature of the lattice and lattice defects have profound effects on the development of the multi-soliton lattice close to the lock-in transition (to another commensurate structure with increased unit cell dimensions) at a lower temperature (say, at $T_{\mathrm{C}}$ ). Introduction of quenched disorder by substitutional impurities at different sites in the lattice will further enhance such pinning effects, and any such perturbations on the phason spectrum introduce a gap in the phason spectrum. The symmetry properties of the dopant play an important role in determining the properties of the IC phases [5], and the impurities are thus characterised as belonging to the symmetry breaking or symmetry non-breaking type, depending on the symmetry they introduce in the free energy of the system (relative to the host). The pinning strength of the dopants can differ widely, leading to the concept of weak or strong pinning [5] depending on the interaction energy of the defect with phasons in relation to the "elastic" energy. Description of these transitions in terms of system-specific microscopic lattice dynamics models, or of semimicroscopic models with competing interactions with nearest and next-nearest neighbours provides a convenient comparison with the known magnetic transitions [6]. Of particular interest in our context is the phenomenological Landau theory [6], wherein the two component (complex) order parameter (describing a one dimensional mass density wave), $Q$, provides a symmetry preserving invariant term (Lifshitz invariant) in the free energy, but which could lead to the minimisation of the free energy, in favourable cases, at an arbitrary point in the Brillouin zone (without any reference to the symmetry of the high temperature phase). This provides us a with a prescription for the onset of the IC modulation near the transition as well as the basis for a description of its development deep in the IC phase. The fluctuations in the atomic displacements due to the IC modulation lead to statistical correlations (in time) in each of the two branches (phason and amplitudon) of the excitation spectrum (particularly within the time window of the magnetic resonance experiments), and hence the corresponding relaxation rates, sensitive to the local spectral densities, provide a very useful tool for obtaining information about 
these dynamic processes. The presence of an inhomogeneous microscopic environment (due to the incommensurability of the medium with respect to the underlying lattice) and hence of a differing static and dynamic environment at different nuclear probes, offers a unique opportunity to probe the two excitation spectra independently by relaxation measurements over the inhomogeneously broadened spectrum [2]. Any microscopic mechanism which affects the power spectrum of these two branches (like the onset of a phason gap, or the development of the so-called Devil's Stair Case (DSC) $[4,7]$ ) now becomes observable through the corresponding observation of the, say, nuclear spin-lattice relaxation rates: $T_{1 \mathrm{~A}}^{-1}$ (mediated by amplitudons) and $T_{1 \phi}^{-1}$ (mediated by phasons). Delineation of these two contributions from detailed measurements on the magnetic resonance spectrum is a fortuitous circumstance that makes this technique very useful, and sometimes unique.

The development of a multi-soliton lattice (MSL) well below $T_{\mathrm{I}}$, the critical soliton density near $T_{\mathrm{C}}$, and the role of defects in affecting the pre-transitional behaviour of the lock-in transition are some of the aspects that were profitably investigated by the NMR techniques $[2,8]$. Similar critical effects near $T_{\mathrm{I}}$ lead to even more curious consequences: the disappearance of the IC order on progressively nearing the transition leads to thermal depinning of the modulation wave critically, and hence there exists a temperature region near $T_{\mathrm{I}}$ where the phason dynamics resembles that of a thermally activated "particle" confined within a finite potential barrier, the height of which is critically temperature dependent. The consequent diffusion-like motion of the modulation wave over the barrier, observed through an equally interesting methodology employing NQR spin echo modulations $[9,10]$, is very unique in terms of the time scales involved (diffusion processes on the scale of $10^{-13}$ to $10^{-15} \mathrm{~cm}^{2} \mathrm{~s}^{-1}$ ). Recent work on this aspect in the presence of different types of impurities (providing differing pinning environments) is discussed below.

This paper introduces the theoretical basis for the use of $\mathrm{NQR}$ technique to investigate different aspects of the IC phases based on the above general ideas, and presents detailed results on the slow-diffusion studies including recent results from our laboratory.

In addition to the extensive investigations on the IC phase, NQR is also used to investigate the role played by the dynamics of the anion $\left(\mathrm{ZnCl}_{4}^{-2}\right)$ in determining the stability of the high temperature (above $T_{\mathrm{I}}$ ) commensurate phase (the so-called para-phase) against the formation of the IC phase below. NQR studies were utilized to understand and correlate the dynamics (torsional motions and the corresponding activation energies) with the paraphase stability, particularly in the presence of impurities at different sites in the lattice [11-13].

\section{NQR in Incommensurate Phases}

\section{Line Shapes and Spin-lattice Relaxation Rates}

The four chlorine nuclei in $\mathrm{Rb}_{2} \mathrm{ZnCl}_{4}$ give rise to three distinct ${ }^{35} \mathrm{ClNQR}$ lines in the intensity ratio of $2: 1: 1$ in the para-phase displaying the chemical inequivalence. At the onset of the IC phase, the lines are inhomogeneously broadened, and the chlorine nucleus with the bond parallel to the direction of modulation, $\mathrm{Cl}(1)$, has a more manageable (less compared to the others) width in the IC phase. Also, since it lies along the IC modulation wave, it is customary to concentrate on the NQR of this chlorine. The two component order parameter $Q$, in the constant amplitude approximation (CAA), is expressed as $\eta \exp (i \phi(z))$, where $\eta$ is a real constant and $\phi(z)$ is a slowly varying function of $z$. The free energy expression can be written as

$$
\begin{aligned}
f(z)= & \frac{1}{2} \mathscr{A}\left(Q^{*} Q\right)+\frac{1}{4} \mathscr{B}\left(Q^{*} Q\right)^{2} \\
& +\frac{1}{6} \mathscr{C}\left(Q^{*} Q\right)^{3}+\frac{1}{2} \mathscr{D}\left(Q^{p}+Q^{*}\right) \\
& -\frac{1}{2} i \sigma\left[Q \frac{\mathrm{d} Q^{*}}{\mathrm{~d} z}-Q^{*} \frac{\mathrm{d} Q}{\mathrm{~d} z}\right] \\
& +\frac{1}{2} \kappa \frac{\mathrm{d} Q}{\mathrm{~d} z} \frac{\mathrm{d} Q^{*}}{\mathrm{~d} z} .
\end{aligned}
$$

The fifth term in (1) is the Lifshitz invariant term (referred to above), and its presence leads to a spatially modulated wave. In the CAA and PWL, the minimum of the above energy occurs in the wave-vector $(\boldsymbol{q})$ space at $q_{0}=\left|\boldsymbol{q}_{c}\right|+\sigma / \kappa$. Here $\boldsymbol{q}_{c}$ is the wave-vector corresponding to the low temperature commensurate phase (below $\left.T_{\mathrm{C}}\right) \cdot q_{0}$ is an arbitrary point whose position in the Brillouin zone is determined by the material coefficients $\sigma$ and $\kappa$. Under CAA, the free energy minimisation corresponds to the Sine-Gordon equation

$$
\kappa \eta^{2}\left[\frac{\partial^{2} \phi}{\partial z^{2}}\right]+\mathscr{D} p \eta^{p} \sin (p \phi)=0
$$

to be satisfied by the phase function.

The excitation spectrum consists of two branches: an acoustic-like excitation (phasons) arising due to the fluctuations of the phase of the displacement profile of the 
modulation wave, with the dispersion relation given by $\omega_{\phi}^{2}=K k^{2}$ in the absence of pinning and an optic-like branch of amplitudons (fluctuations of the amplitude of the displacement) with a relation $\omega_{\mathrm{A}}^{2}=2 a\left(T_{\mathrm{I}}-T\right)+K k^{2}$. $\mathrm{NQR}$ being sensitive to the local electric field gradients experienced by the probe nucleus, shift in frequency at any point $z$ on the modulation wave can be expressed in terms of the local displacement $u(z)=A \cos (\phi(z))$ as $v=v_{0}+v_{1} \cos \phi+v_{2} \cos ^{2} \phi+\ldots$. In the "local case" (the wave length of the modulation wave is much greater than the effective length scale of the interaction governing the resonance frequency) the line shape, defined by $f(v)=$ Const./( $\mathrm{d} v / \mathrm{d} \phi)$, is broad with weak singularities (their number and position depending on the relative strengths of the linear and quadratic terms in the above expression for the frequency $v$ ). It is possible to calculate the spinlattice relaxation rate of the quadrupolar spins located at any point on the modulation wave as

$$
\begin{aligned}
& T_{1 \mathrm{Q}}^{-1}=X^{2}\left(T_{1 \mathrm{~A}}^{-1}\right)+\left(1-X^{2}\right)\left(T_{1 \phi}^{-1}\right), \\
& \text { where } X=\cos (\phi(z)) .
\end{aligned}
$$

This facilitates the separation of contributions from the different excitations to the observed relaxation rate. In the PWL, the phason- and amplitudon-gaps $\left(\Delta_{\phi}\right.$ and $\Delta_{\mathrm{A}}$, respectively) can be related to the relaxation rates as $\left(T_{1 \phi} / T_{1 \mathrm{~A}}\right)=\left(\Delta_{\phi} / \Delta_{\mathrm{A}}\right)$, permitting estimates of phason gaps from the two relaxation rates.

\section{Coupling with Impurities}

Impurities introduce disorder in the structure, and considering only the defects which couple to the phase of the IC modulation wave (and are randomly quenched), one can introduce an additional free energy

$$
\Delta F_{\text {imp }}=-V \sum_{i} \rho^{m}\left(r_{i}\right) \cos \left(m \phi\left(r_{i}\right)+\delta_{m}\right),
$$

where $m$, the symmetry parameter of the impurity, is an integer.

Random-field impurities act as random fields on the commensurate order-parameter, discriminating among the $n$ possible commensurate domains. They can be realized by coupling to the order parameter as $Q^{m},(m<n$ in the above equation). Random-interactions, also referred to as random-potentials, do not break the symmetry of the functional (i.e. $m=n$ in the above equation), but change the anisotropy strength $(\gamma \rightarrow \gamma+\Delta \gamma)$ locally.

$T$ and $n_{i}$ (impurity concentration) dependence the phason gap is

$$
\Delta_{\phi} \propto\left(T_{\mathrm{I}}-T\right)^{\beta(m-2)} \sqrt{n_{i}} .
$$

The development of the phason gap near $T_{\mathrm{I}}$ (as evidenced by the $T_{1 \phi}$ ) thus provides a clue to the nature of the pinning centre. The impurities are further distinguished as of the strong or weak pinning type, depending on their pinning strength, and lead to temperature independent or dependent phason gaps, respectively.

In the MSL regime, there is a possibility that the system undergoes a series of first order transitions among structures which have regular (commensurate) soliton lattices. Such phenomena (taking place near $T_{\mathrm{C}}$, where the soliton width becomes comparable with the lattice spacing) are referred to as DSC, and in certain favourable cases (including the presence of induced disorder) are observable as steps in the phason mediated relaxation rates.

\section{Slow Diffusion-like Dynamics near $T_{\mathrm{I}}$}

The dynamics of the IC modulation wave (IMW) near $T_{\mathrm{I}}$ is influenced by two competing processes: (i) pinning of the phason mode due to a finite, critically temperature dependent, and impurity induced gap $\Delta_{\phi}$, and (ii) thermally induced fluctuations of the modulation in the presence of these barriers. The random distribution of these pinning centres with random pinning strength destroys the harmonic character of the thermally induced motion of the IMW. The wave can surmount the pinning barrier momentarily by thermal excitation before it is depinned again on losing the energy to the other degrees of freedom. Since this thermally activated depinning is a stochastic process, the resultant motion (of the IMW, which corresponds to the collective motion of the aggregates of the molecules between the pinning centres), would be random and could be diffusion like. Thermal fluctuations of the wave in the vicinity of $T_{\mathrm{I}}$ have been studied earlier $[14,15]$. Clear evidence of the diffusional character of this dynamics has been provided by the recent work based on NQR spin-echo modulation $[9,10]$.

In NMR studies, the spin echo attenuation in the presence of a field gradient is given by $[2,16]$

$$
M(2 \tau)=M_{0} \exp \left[-\left(\frac{2 \tau}{T_{2 \mathrm{Q}}}+D\left(\gamma^{0} \frac{\partial H}{\partial z}\right)^{2} \frac{2 \tau^{3}}{3}\right)\right]
$$

Here $T_{2 \mathrm{Q}}$ is the spin-spin relaxation time, $\gamma^{0}$ the gyromagnetic ratio of the probe, and $\tau$ the spacing between the rf pulses in a two-pulse experiment. The distinguishing feature of this attenuation is the exponential variation with a cubic power of time. The diffusion coefficient $D$ can be computed from the observed attenuation, provided the magnetic field gradient applied is known. The magnitude of this gradient puts the lower limit on the 
slowness of the diffusive process under study. The achievable gradients in the NMR case can measure $D$ values up to a lower limit of $10^{-9} \mathrm{~cm}^{2} \mathrm{~s}^{-1}$. A very similar situation can be envisaged even in the pure $\mathrm{NQR}$ case, provided one could have a calibrated gradient of the EFG's themselves. While it is next to impossible to generate and calibrate such practically useful gradients from external sources for NQR purposes, IC systems very interestingly do provide such calibrated gradients of the EFG. The location of the weak singularities arising from the extrema of the modulation wave provide such a bench-marking. What is even more attractive is the fact that such gradients of EFG's are so powerful (much more than the laboratory generated magnetic field gradients), that the experiment becomes sensitive to $D$ values smaller than the conventional limit by many orders. In principle thus the IC phase provides a natural calibrated spatial inhomogeneity useful for measurements on diffusion-like motions through NQR spin echo measurements. The onset of thermal depinning near $T_{\mathrm{I}}$, leading to very slow motions of the IMW, affect the phase coherence of a probing NQR nucleus in a spin echo measurement much the same way as the nucleus would have experienced if it were to diffuse over the modulation wave. This fascinating circumstance provides a very convenient and unique technique to probe very slow motions near the incommensurate phase transition via NQR or quadrupole perturbed NMR [10]. The basic difference in the treatment of this problem, in comparison with the standard measurements with NMR based on gradient methods, arises from the fact that the space-frequency encoding relationship is (i) not linear and (ii) periodic. The corresponding Bloch-Torrey equation needs to be solved in the presence of a periodic gradient. While exact solutions do exist for such equations [10], they are intractable, and it is useful to consider only short-scale motions for the present purpose (the justification for such a treatment being provided only $a$ posteriori). Here, only motions with rms amplitudes smaller than the wave length of the IC phase (about $2.8 \mathrm{~nm}$ in $\mathrm{Rb}_{2} \mathrm{ZnCl}_{4}$, for example) are considered.

Following Papavassiliou et al. [10] the variation of the echo amplitude can be written as

$$
M(2 \tau)=M_{0} \exp \left[-\left(\frac{2 \tau}{T_{2}}+D_{\mathrm{f}}(\nabla \omega)^{2} \frac{2 \tau^{3}}{3}\right)\right],
$$

where $(\nabla \omega)^{2}$-represents the square effective gradient (of the EFG) at the position $z$. For non-linear variations in the gradient of the frequency, it is more convenient to define a frequency dependent diffusion coefficient $D_{\mathrm{f}}$. In the short-scale limit the variation of the resonance fre- quency with distance, $\omega(z)$, can be approximated by a linear or quadratic term, appropriately. In the case of pure $\mathrm{Cl} \mathrm{NQR} \mathrm{in} \mathrm{the} \mathrm{above} \mathrm{system,} \mathrm{due} \mathrm{to} \mathrm{the} \mathrm{high} \mathrm{tempera-}$ ture mirror symmetry in the para-phase, quadratic approximation of $\omega(z)$ (particularly very near $T_{\mathrm{I}}$ ) is adequate. The resultant model suggests [10]: (i) the diffusion coefficient $D$ depends on the position of the probe on the IMW, (ii) the diffusion coefficient is maximum at the centre of the spectrum and should represent the true diffusion coefficient, and (iii) the apparent diffusion as seen at the edge singularities of the spectrum (corresponding to the extrema in the modulation wave) is negligible due to negligible changes in the frequency near these extrema and hence due to negligible dephasing effects despite motion. The authors [10] provide a detailed account of their findings in a pure crystalline sample of $\mathrm{Rb}_{2} \mathrm{ZnCl}_{4}$ and show clearly the presence of extremely slow diffusive motions $\left(D \sim 5 \times 10^{-13} \mathrm{~cm}^{2} \mathrm{~s}^{-1}\right)$ taking place in the vicinity $\left(T_{\mathrm{I}}-T \sim 0.5 \mathrm{~K}\right)$ of the incommensurate transition. This corresponds to an average value of the step size, $z_{\mathrm{av}}$, of $0.3 \mathrm{~nm}$ (about $10 \%$ of the IC wave length). The case of quadrupole perturbed NMR spin echoes of ${ }^{39} \mathrm{~K}$ in $\mathrm{K}_{2} \mathrm{SeO}_{4}$ is also discussed in detail in this reference.

\section{Representative Experimental Results}

This section summarises representative results from our laboratory on some of the aspects of the dynamics discussed above. The measurements were carried out on a number of polycrystalline samples of $\mathrm{Rb}_{2} \mathrm{ZnCl}_{4}(\mathrm{RZC})$ doped with impurities substituted at three different sites, in some cases with varying concentration. These results are always compared with the corresponding measurements with nominally pure polycrystalline RZC samples. The samples investigated are: $\mathrm{Rb}_{2} \mathrm{ZnCl}_{4} ;\left[\mathrm{Rb}_{1-x}\left(\mathrm{NH}_{4}\right)_{x}\right]_{2}$ $\mathrm{ZnCl}_{4}$ for $x=0.07 ;\left[\mathrm{Rb}_{1-x}(\mathrm{TMA})_{x}\right]_{2} \mathrm{ZnCl}_{4}$ (where TMA = $\mathrm{N}\left(\mathrm{CH}_{3}\right)_{4}$ for $x=0.03,0.08$ and $0.12 ; \mathrm{Rb}_{2}\left(\mathrm{Zn}_{1-x} \mathrm{Cu}_{x}\right) \mathrm{Cl}_{4}$ for $x=0.03$ and $0.05 ; \mathrm{Rb}_{2}\left(\mathrm{Zn}_{1-x} \mathrm{Cd}_{x}\right) \mathrm{Cl}_{4}$ for $x=0.03$ and $0.05 ; \mathrm{Rb}_{2}\left(\mathrm{Zn}_{1-x} \mathrm{Hg}_{x}\right) \mathrm{Cl}_{4}$ for $x=0.03$ and 0.05 ; $\mathrm{Rb}_{2} \mathrm{Zn}\left(\mathrm{Cl}_{1-x} \mathrm{Br}_{x}\right)_{4}$ for $x=0.01$ and 0.03 . The measurements were made on a home-made pulsed NQR spectrometer with a suitable temperature variation facility. The weak singularities on the inhomogeneously broadened NQR spectrum were detected by applying soft pulses (a $\pi / 2$ pulse width of about $100 \mu \mathrm{s})$ and by scanning the broad spectrum. Measurements on the variation of locations of the singularities as well as the relaxation times were carried out by placing the soft pulse centred at the 

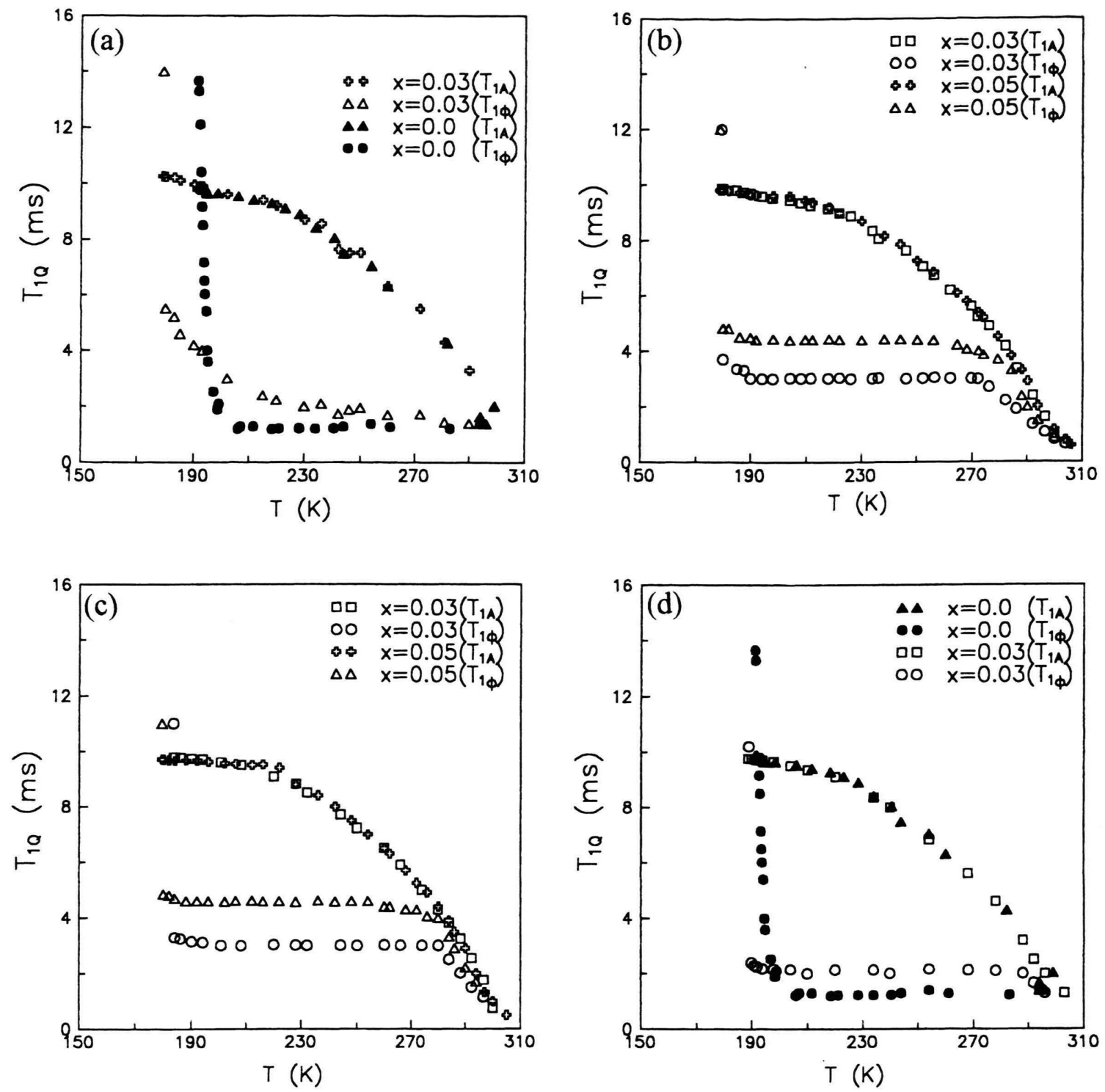

Fig. 1. Variation of the spin-lattice relaxation times $T_{1 \mathrm{~A}}$ and $T_{10}$ with temperature in incommensurate phases: (a) $\mathrm{Rb}_{2} \mathrm{ZnCl}$ and $\mathrm{Rb}_{2} \mathrm{Zn}\left(\mathrm{Cl}_{1-x} \mathrm{Br}_{x}\right)_{4}$ for $x=0.03$; (b) $\mathrm{Rb}_{2}\left(\mathrm{Zn}_{1-x} \mathrm{Cd}_{x}\right) \mathrm{Cl}_{4}$ for $x=0.03$ and 0.05 ; (c) $\mathrm{Rb}_{2}\left(\mathrm{Zn}_{1-x} \mathrm{Hg}_{x}\right) \mathrm{Cl}_{4}$ for $x=0.03$ and 0.05 ; (d) $\mathrm{Rb}_{2}\left(\mathrm{Zn}_{1-x} \mathrm{Cu}_{x}\right) \mathrm{Cl}_{4}$ for $x=0.03$. Substitution at $\mathrm{Cl}$ site, Fig. a, shows weak pinning, and substitutions at the $\mathrm{Zn}$ site, Figs. $\mathrm{b}-\mathrm{d}$, exhibit strong pinning.

corresponding positions. Extensive signal averaging was found necessary in some cases due to poor signal-to-noise ratio values.

\section{Characterisation of Pinning Centres}

Measurements on the phason mediated spin-lattice relaxation $\left(T_{1 \phi}\right)$ with different dopants in the RZC system provide a contrasting comparison [17]. Substitution at the $\mathrm{Rb}$ (with $\mathrm{K}, \mathrm{NH}_{4}$, TMA, or $\mathrm{Cs}$ ) or $\mathrm{Cl}$ (with $\mathrm{Br}$ ) sites have always resulted in a temperature dependent relaxation time, indicating a temperature dependent pinning effect, and this would correspond to a weak pinning case (for example, Figure 1(a)). On the other hand, substitution at the $\mathrm{Zn}$ site seems to induce always a strong pinning, as evidenced in Figs. 1(b)-(d) (with $\mathrm{Cu}, \mathrm{Cd}$, and 
Table 1.

\begin{tabular}{|c|c|c|c|c|c|c|c|c|c|}
\hline Compound & $\begin{array}{l}T_{1} \\
(\mathrm{~K})\end{array}$ & $\begin{array}{l}\Delta T \\
(\mathrm{~K})\end{array}$ & $\begin{array}{l}T_{2 \mathrm{Q}} \\
(\mu \mathrm{S})\end{array}$ & $\begin{array}{l}D_{(\mathrm{f}-\max )} \\
\mathrm{cm}^{2} / \mathrm{s}\end{array}$ & $\begin{array}{l}t \\
(\mu \mathrm{s})\end{array}$ & $\begin{array}{l}\Delta \omega \\
(\mathrm{kHz})\end{array}$ & $\lambda$ & $\begin{array}{l}x \\
(\mathrm{~nm})\end{array}$ & $m$ \\
\hline $\mathrm{Rb}_{2} \mathrm{ZnCl}_{4}$ & 302 & 0.5 & 550 & $1.12 \mathrm{E}-12$ & 1550 & 3.82 & 2.97 & 0.58 & 6 \\
\hline$\left[\mathrm{Rb}_{1-x}(\mathrm{TMA})_{x}\right]_{2} \mathrm{ZnCl}_{4}, x=0.08$ & 300 & 0.5 & 540 & $6.01 \mathrm{E}-15$ & 1945 & 14.25 & 1.25 & 0.048 & 5 \\
\hline$\left[\mathrm{Rb}_{1-x}(\mathrm{Cs})_{x}\right]_{2} \mathrm{ZnCl}_{4}, \quad x=0.005$ & 298 & 0.5 & 545 & $3.05 \mathrm{E}-13$ & 1350 & 5.81 & 3.27 & 0.29 & 6 \\
\hline$\left[\mathrm{Rb}_{1-x}\left(\mathrm{NH}_{4}\right)_{x}\right]_{2} \mathrm{ZnCl}_{4}, \quad x=0.07$ & 305 & 0.5 & 540 & $3.38 \mathrm{E}-13$ & 1740 & 3.66 & 3.43 & 0.34 & 5 \\
\hline $\mathrm{Rb}_{2}\left(\mathrm{Zn}_{1-x} \mathrm{Cd}_{x}\right) \mathrm{Cl}_{4}$ & 305 & 0.5 & 360 & $4.88 \mathrm{E}-13$ & 1350 & 6.62 & - & 0.36 & 4 \\
\hline $\mathrm{Rb}_{2}\left(\mathrm{Zn}_{\mathrm{l}-x} \mathrm{Hg}_{x}\right) \mathrm{Cl}_{4}$ & 308 & 0.7 & 340 & $7.11 \mathrm{E}-13$ & 1350 & 1.86 & - & 0.44 & 4 \\
\hline $\mathrm{Rb}_{2} \mathrm{Zn}\left(\mathrm{Cl}_{1-x} \mathrm{Br}_{x}\right)_{4}$ & 293 & 0.4 & 530 & $3.42 \mathrm{E}-13$ & 1540 & 7.05 & - & 0.32 & 6 \\
\hline
\end{tabular}

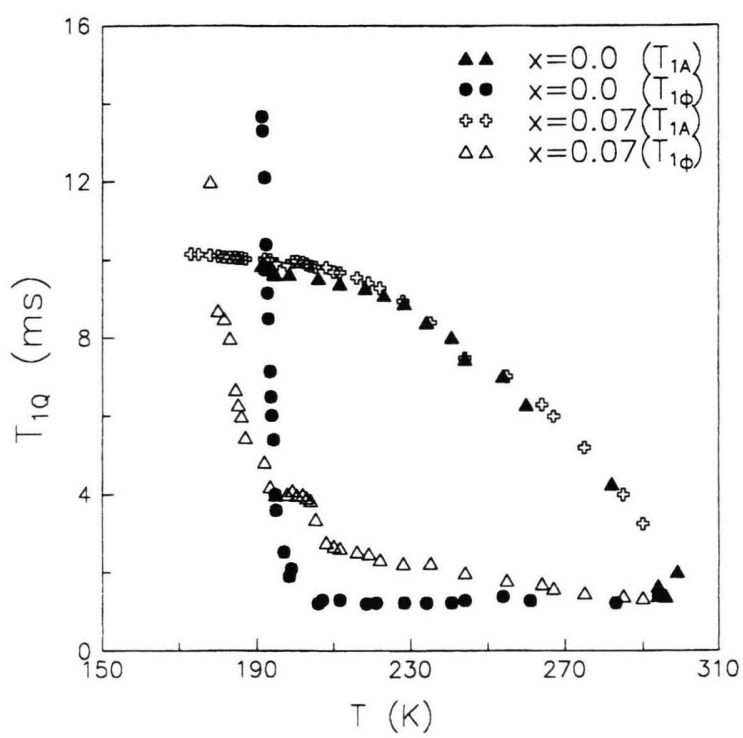

Fig. 2. Variation of the spin-lattice relaxation times $T_{1 \mathrm{~A}}$ and $T_{1 \phi}$ with temperature in the incommensurate phase of $\left[\mathrm{Rb}_{1-x}\left(\mathrm{NH}_{4}\right)_{x}\right]_{2}$ $\mathrm{ZnCl}_{4}$ for $x=0.07$. The phason contribution shows the formation of the Devil's Stair Case (DSC).

Hg substitutions). This seems to be the first observation of a strong pinning in RZC and seems to result whenever an impurity is embedded at the $\mathrm{Zn}$ site. Using the (5) and the relation between $T_{1 \phi}$ and $\Delta_{\phi}$, the symmetry parameter $m$ for the different dopants is evaluated (Table 1), and this permits classification of the defects as being of random-field type or random-potential type.

\section{Induced Devil's Stair Case in RZC}

Substitution with TMA at the Rb site (in the RZC system) seems to be particularly interesting and intriguing [18]. It is known that certain impurities could make the DSC observable over a temperature range (for exam-

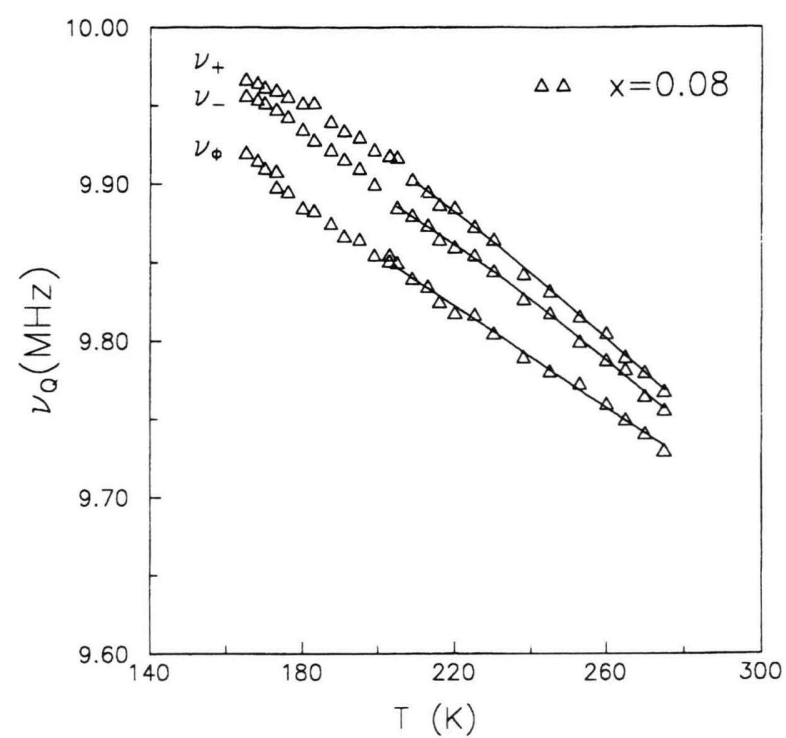

Fig. 3. Variation of the position of singularities in $\left[\mathrm{Rb}_{1-x}\right.$ $\left.(\mathrm{TMA})_{x}\right]_{2} \mathrm{ZnCl}_{4}$ for $x=0.08$.

ple $\mathrm{NH}_{4}$ substitution with $x=0.08$, Figure 2). Since the coupling of the impurity is through phasons, such steps are expected to be observed in the variation of $T_{1 \phi}$ with the temperature [19]. It may be noted that the amplitudon branch is not affected by this stair case. However TMA substitution seems to lead to a very different behaviour. To start with, the three singularities $\left(v_{+}, v_{-}\right.$, and $v_{\phi}$ ) in the TMA doped system (with $x=0.08$ ) do not behave (and diverge) as the system is cooled away from the $T_{\mathrm{I}}$ (Figure 3). Their expected critical behaviour deviates below about $210 \mathrm{~K}$, and the $v_{+}$and $v_{-}$lines in fact start coalescing. The splitting between these two singularities originating from the small linear term in the expression for $v$ decreases corresponding to a qualitative change in 


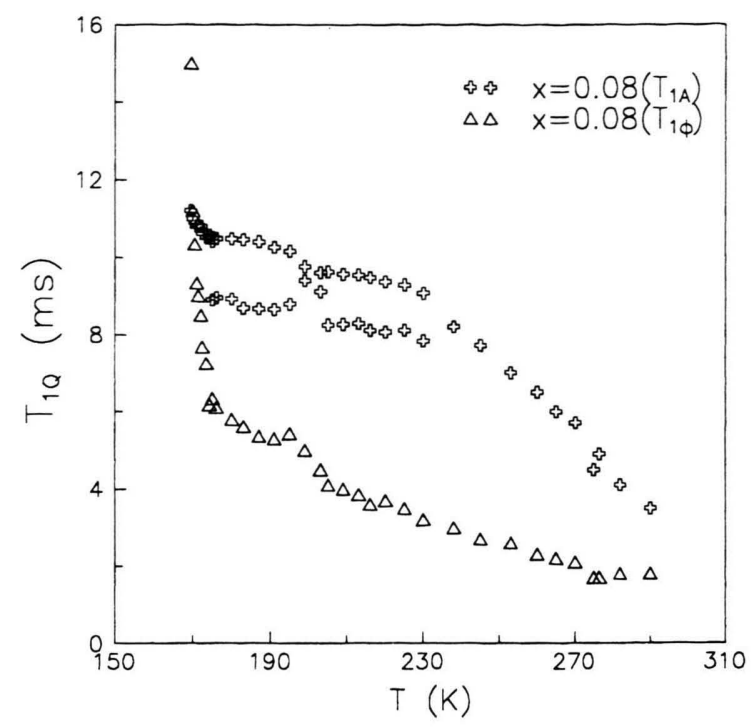

Fig. 4. Variation of the spin-lattice relaxation times $T_{1 \mathrm{~A}}$ and $T_{10}$ with temperature in the incommensurate phase of $\left[\mathrm{Rb}_{1-x}\right.$ (TMA) $\left.]_{2}\right]_{2 n C l}$ for $x=0.08$. The phason and amplitudon contributions show a correlated step-like structure.

the structure of the modulation wave. Equally interesting is the observation on the relaxation behaviour of the two singularities, i.e., $T_{1 \mathrm{~A}}$ and $T_{1 \phi}$ (Figure 4). Here, below about $220 \mathrm{~K}$, two plateau regions are observed in the $T_{1 \mathrm{~A}}$ and $T_{1 \phi}$ variations. These temperature ranges coincide with the observed anomalies in the position of the singularities (of $v_{+}$and $v_{-}$). The observation of plateau regions in $T_{1 \mathrm{~A}}$ is rather puzzling, particularly in the case of pure NQR. Further analysis of the data indicates that the magnetisation recovery corresponding to the $T_{1 \mathrm{~A}}$ measurement in the plateau regions is non-exponential and requires two time constants for a satisfactory fit to the data. And the non-exponentiality is absent outside the plateau regions, including the region in between the two plateau regions. The magnetisation recovery corresponding to $T_{1 \phi}$, on the other hand, shows no such non-exponential behaviour within the experimental error. The two split curves (in a given plateau) of $T_{1 \mathrm{~A}}$ correspond to the two best fit relaxation times. These features are even more pronounced with a higher concentration $(x=0.12)$. There could be several possibilities leading to such curious observations, including: the possibility of coupling between the amplitudon and phason branches (cross-relaxation); the development of non-local behaviour below a certain temperature for some reason; and of course the conjecture (backed by the frequency domain data) that perhaps the system is undergoing more subtle changes with temperature like a sequence of incommensurate phases with a multi-dimensional IC modulation. This last suggestion can in principle account for the observations. Coupled with the facts that (i) the pure (TMA) ${ }_{2} \mathrm{ZnCl}_{4}$ itself shows evidence of DSC (even though only through $T_{1 \phi}$ ), (ii) its own IMW is in a different direction with a different $q_{0}$ value, and (iii) it represents a weak symmetry breaking $(m=5)$ impurity in the RZC system, this suggestion perhaps merits further investigation. Details of this work are to appear elsewhere [18].

\section{Slow Diffusion-like Motion of Incommensurate Modulation Wave}

The recent work initiating measurements on very slow processes involving aggregates of molecules in crystalline RZC provides both a viable methodology as well as motivation to look for effects of externally induced disorder on the thermal depinning effects. In this context spin echo attenuation measurements were taken up in all the doped systems near the respective $T_{\mathrm{I}}$ 's. The details of the experiment and the new results obtained in polycrystalline samples are reported here. The amplitude of the NQR spin-echo, formed through the application of a $\pi / 2-\tau-\pi$ sequence, has been measured as a function of the inter-pulse delay $\tau$. The high frequency line corresponding to $\mathrm{Cl}(1)$ was chosen for this purpose. The temperature range covered in each case extended over about $10 \mathrm{~K}\left(T_{\mathrm{I}} \pm 5 \mathrm{~K}\right)$.

The data were collected with a minimum of 1024 averages, and the spin-echo modulation envelopes thus obtained were analyzed using (7) through a non-linear least square procedure resulting in best fit values for $T_{2 \mathrm{Q}}$ and $D_{\mathrm{f}}$ for each spin-echo envelope. The calibration of the space-frequency relationship is obtained by extrapolating the observed splittings between the two edge singularities over a temperature range very near the $T_{\mathrm{I}}$ with a known power law behaviour (Figure 5). It is observed that just above the corresponding $T_{\mathrm{I}}$ value (in the respective para-phases) all the samples show a single exponential behaviour, yielding the $T_{2 \mathrm{Q}}$ values for the spin-echo decay. It is also seen that the decays obey a simple $T_{2 \mathrm{Q}}$ variation about 5 degrees below $T_{\mathrm{I}}$, indicating that the interesting diffusive motions in all the samples are taking place just within the 5 degrees below $T_{\mathrm{I}}$ in the polycrystalline samples, compared to about 10 degrees in the crystalline pure RZC sample. A variation of the profile of the spin-echo attenuation with temperature in polycrystalline sample is presented in Figure 6. 


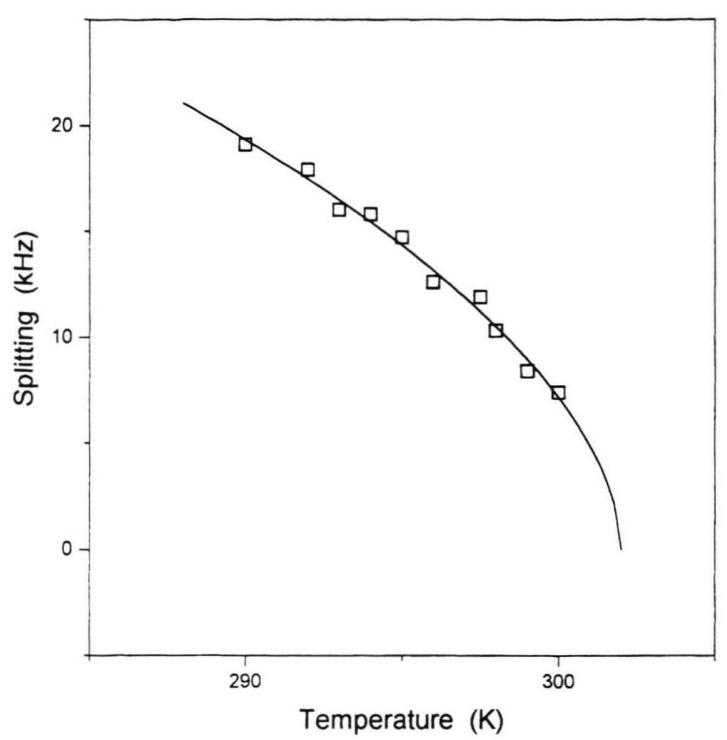

Fig. 5. Variation of the position of the edge singularities in nominally pure polycrystalline $\mathrm{Rb}_{2} \mathrm{ZnCl}_{4}$ near $T_{\mathrm{I}}$. The solid curve is the best fit curve.

The model [10] developed for the quadratic dependence of the frequency on the positional coordinate predicts that the true diffusion coefficient, providing a quantitative dynamic parameter connected with the collective motions of the ensemble of molecules, is observable in the centre of the broadened spectrum. Also, within the small temperature range available for measurements on these powder samples, it is not possible to extract information on the frequency dependent diffusion constant $D_{\mathrm{f}}$ over the spectrum. So the experiments were carried out by keeping the rf frequency at the centre of the spectrum and the measurements were made with soft pulses. These results provide an average diffusion coefficient $D_{\text {eff }}$, comparable to the true value. The value of $D_{\text {eff }}$ obtained at about $0.5 \mathrm{~K}$ below the $T_{\mathrm{I}}$ is $1.1 \times 10^{-12} \mathrm{~cm}^{2} \mathrm{~s}^{-1}$ in a nominally pure polycrystalline RZC sample, while the reported value under identical conditions in a pure crystalline RZC sample is $5.3 \times 10^{-13} \mathrm{~cm}^{2} \mathrm{~s}^{-1}$ (about half of the value measured in the present case). The reasonable agreement (in terms of the order of magnitude of the coefficient) in powder and crystalline samples is taken as an acceptable basis for comparing the results of such measurements on all other doped samples. The variation of $D_{\text {eff }}$ in a few representative systems are shown in Figure 7. It is immediately observed that the general effect of introducing disorder in the medium is to reduce the mobility of the modulation wave and hence a consequent
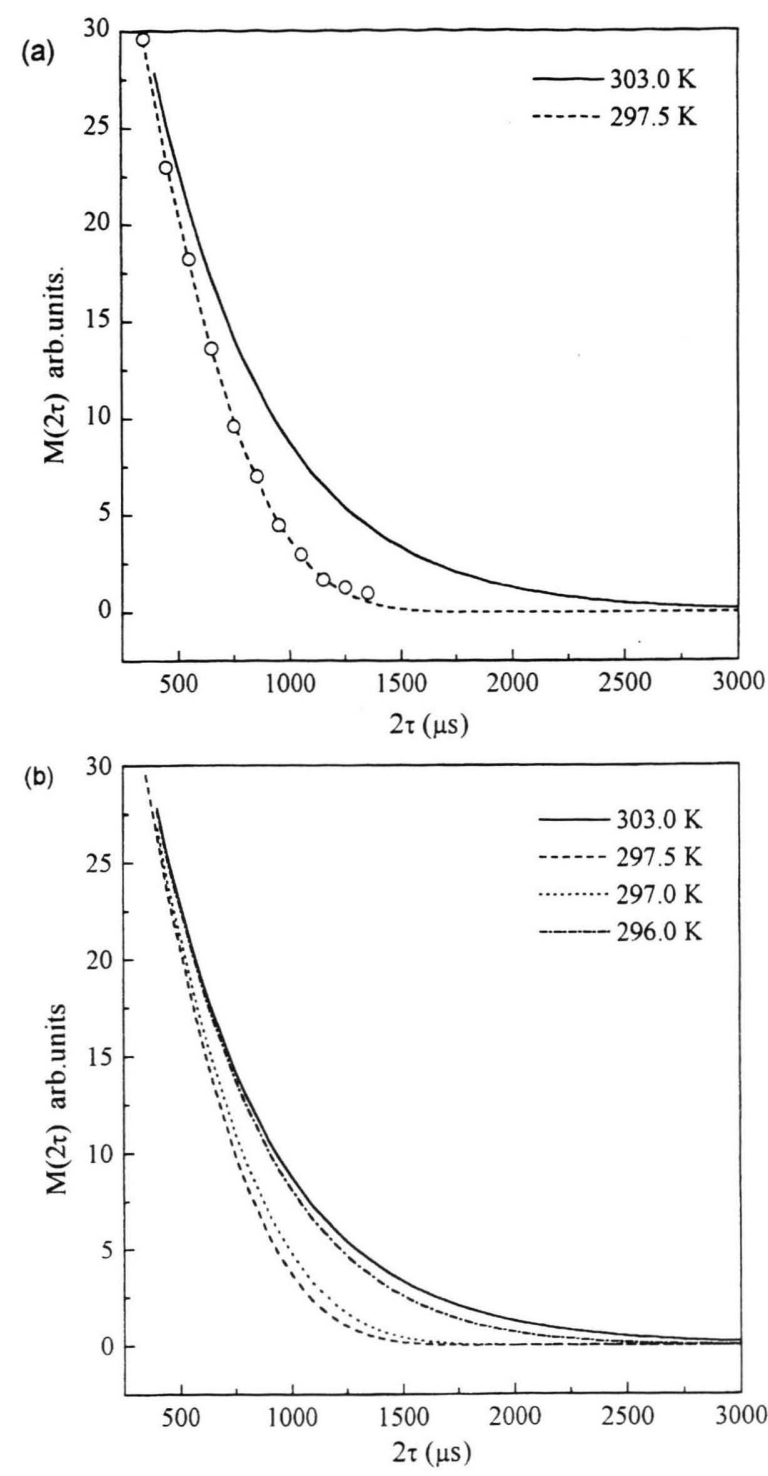

Fig. 6. (a) Typical spin-echo decay with (---) and without (-) diffusion at two temperatures with data shown in one case $(0)$. The sample is $\left(\mathrm{Rb}_{1-x} \mathrm{Cs}_{x}\right)_{2} \mathrm{ZnCl}_{4}(x=0.005)$. (b) Best fit curves to the spin-echo decays as a function of temperature.

reduction in the diffusion coefficient. Interestingly, in all the systems studied, with the exception of the TMA doped system, the $D_{\text {eff }}$ values are reduced to about 30 to $40 \%$ of the corresponding value for the pure (polycrystalline) RZC sample (Table 1). The TMA doped sample, however, shows relatively very slow (about $5 \%$ ) diffusion ( $D_{\text {eff }}$ is $6.0 \times 10^{-15} \mathrm{~cm}^{2} \mathrm{~s}^{-1}$ at $0.5 \mathrm{~K}$ below the corresponding $T_{\mathrm{I}}$ ). This is a curious observation, and the one thing that distinguishes the TMA from the others substituents is the 

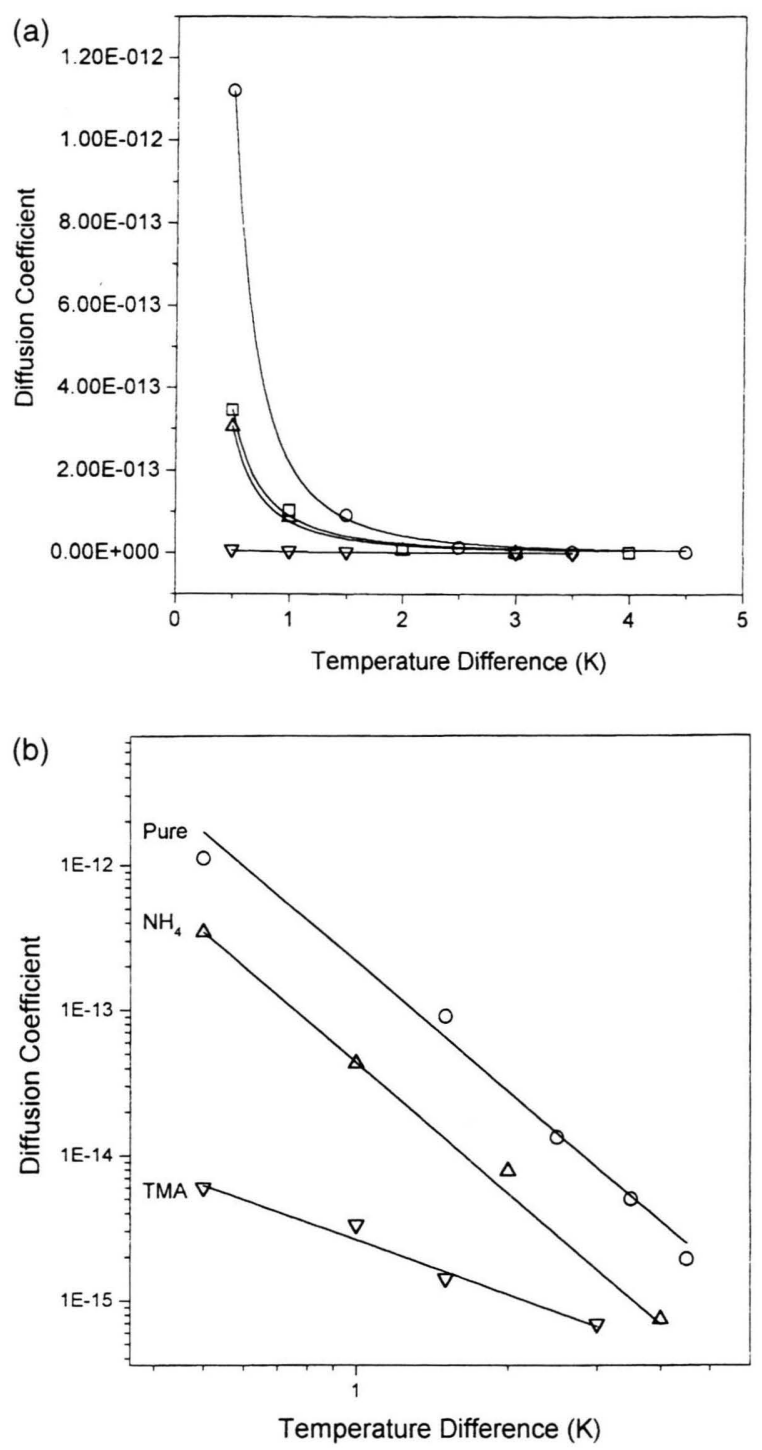

Fig. 7. (a) Variation of diffusion coefficient $\left(D_{\text {eff }}\right)$ with temperature in different samples $\left[\mathrm{Rb}_{2} \mathrm{ZnCl}_{4}(\mathrm{O}) ;\left(\mathrm{Rb}_{1-x}\left(\mathrm{NH}_{4}\right)_{x}\right)_{2}\right.$ $\mathrm{ZnCl}_{4}(\square) ;\left(\mathrm{Rb}_{1-x} \mathrm{Cs}_{x}\right)_{2} \mathrm{ZnCl}_{4}(\triangle)$; and $\left(\mathrm{Rb}_{1-x}(\mathrm{TMA})_{x}\right)_{2}$ $\mathrm{ZnCl}_{4}(\nabla)$. The solid lines are the best fit theoretical curves. (b) Variation of $D_{\text {eff }}$ as in (a), but plotted on a logarithmic scale to show the temperature variation in TMA sample better.

many degrees of freedom it has due to the presence of mobile methyl groups, somehow effectively pinning the modulation wave and reducing its mobility.

In order to account for the observed critical temperature dependence of the diffusion coefficient, one considers the short range interaction between the modulation wave $u\left(r_{i}\right)$ and the impurities or defects. It can be ex- pressed in the plane wave approximation and the strong coupling limit as [20]:

$$
H_{\mathrm{I}}=-\sum_{i} W_{i} u\left(r_{i}\right) \text {, }
$$

where the sum is over all defects. $W_{i}$ is a coupling constant quantifying the impurity pinning strength, and

$$
u(r)=A_{0} \cos \left(k_{\mathrm{I}} r+\phi(r)\right) .
$$

$A_{0}$ is proportional to $\left(T_{\mathrm{I}}-T\right)^{\beta}$ and $\phi(r) \neq 0$ describes the influence of impurities on the phase of the modulation wave. As a result of the strong coupling with impurities, $\phi$ adjusts itself on the impurity sites so that the displacement is maximal, i.e., $u(r)=A_{0}$ and $k_{\mathrm{I}} \cdot r_{i}+\phi\left(r_{i}\right)=2 \pi m$; $m=1,2,3, \ldots$. The sliding of the modulation wave can be considered to be activated over a barrier $U(A)=2 W A^{v}$, where $U(A)$ depends on the $v^{\text {th }}$ power of $A \propto\left(T_{1}-T\right)^{\beta}$. If we define a critical barrier height $E_{0}$ for motional narrowing, then only defects for which $2 W A^{v}>E_{0}$ will be effective in impeding motional narrowing.

Assuming for simplicity that the distribution of defects is of the form $[10,14,15]$

$$
n(W)=b W^{-\gamma},
$$

we obtain the effective number of such pinning defects per unit volume as

$$
\begin{aligned}
n_{\text {eff }}= & \int_{E_{0} / 2 A_{0}}^{\infty} n(W) \mathrm{d} W \propto \frac{1}{\gamma-1}\left(\frac{E_{0}}{2 A_{0}}\right)^{1-\gamma} \\
& \propto\left(T_{\mathrm{I}}-T\right)^{\beta(\gamma-1)},
\end{aligned}
$$

and the mean distance between pinning defects as

$$
l_{0}=\frac{1}{3 n_{\mathrm{eff}}^{1 / 3}} \propto \frac{1}{\left(T_{\mathrm{I}}-T\right)^{\beta(\gamma-1) / 3}},
$$

increasing rapidly as $T \rightarrow T_{\mathrm{I}}$. At this limit the spacing between pinning defects becomes rather large, and in all cases only a single motionally averaged line should remain. Consequently, thermally induced floating of the pinned modulation wave becomes indistinguishable from uniform sliding of the modulation wave (as $T \rightarrow T_{\mathrm{I}}$ ). Such random motions of the modulation wave can be described by an effective diffusion constant $D_{\text {eff }}=l^{2} / \tau_{D}$, where $l$ is the average step of the random-walk and $\tau_{D}$ is the thermally activated transition time per step $\left(\tau_{D}=\tau_{D_{0}}\right.$ $\exp [U(A) / k T])$. Assuming that $l$ is connected to the impurity density as $l(W) \propto n(W)^{-1 / 3}, D_{\text {eff }}$ has a critical temperature dependence as $D_{\text {eff }} \propto\left(T_{\mathrm{I}} \rightarrow T\right)^{-\lambda}$ where $\lambda=v \beta(1-\gamma / 3)$. Here $\lambda$ reflects the nature of the coupling of the impurity to the modulation wave. 
The data on $D_{\text {eff }}$ as a function of temperature were analysed using the above model of thermal depinning in the presence of quenched impurities. The data in the presence of impurities at $\mathrm{Zn}$ and $\mathrm{Cl}$ sites as a function of temperature near $T_{\mathrm{I}}$ turned out to be rather noisy, and so a reliable estimate of the exponent $\lambda$ could not be made for these cases. Its values for the other cases are available and quoted in the Table 1 (Figure 7).

There were earlier reports on the magnitude of this exponent in single crystal studies. The value of $\lambda$ was quoted to be 3.49 for measurements on the high frequency singularity, and 1.8 for the low frequency one [9(a)]. It was concluded that this variation suggested a more elaborate model for this motion. Subsequent work [10] with an improved theoretical basis led to an exponent value of 1.31 only. In view of the sensitivity of the exponent on the position of the measured line, and the fact that the samples in the present study are polycrystalline in nature, the absolute value of the exponent is perhaps not so relevant as the variation of the exponent with substitution (all the measurements on different samples were done at identical experimental conditions). Here again, TMA substitu-

[1] H. Z. Cummins, Phys. Rep. 185, 211 (1992).

[2] R. Blinc, P. Prelovsek, V. Rutar, J. Seliger, and S. Zumer, in Incommensurate Phases in Dielectrics, Eds: R. Blinc and A. P. Levanyuk, Academic Press, New York 1986, Vol. 1, p. 143; R. Blinc, Phys. Rep. 79, 331 (1981).

[3] W. L. Mac Millan, Phys. Rev. B14, 1496 (1976).

[4] P. Bak and V. L. Pokrovsky, Phys. Rev. Lett. 47, 958 (1981).

[5] P. Prelovsek, Phase Trans. 11, 203 (1988).

[6] T. Janssen, in Incommensurate Phases in Dielectrics, Eds: R. Blinc and A. P. Levanyuk, Academic Press, New York 1986, Vol. 1, p. 67; D. G. Sannikov ibid, Vol. 1, p. 43.

[7] S. Aubry, in Solitons and Condensed Matter Physica, Eds.: A. R. Bishop and T. Schneider, Springer-Verlag Berlin 1978 , p. 26.

[8] J. Dolinsek and R. Blinc, J. Phys.: Condensed Matter 21, 705 (1988).

[9] a) G. Papavassiliou, A. Leventis, F. Milia, and J. Dolinsek, Phys. Rev. Lett. 74, 2387 (1995). - b) D. C. Ailion and J. A. Norcross, Phys. Rev. Lett. 74, 2383 (1995).

[10] G. Papavassiliou, M. Fardis, A. Leventis, F. Milia, J. Dolinsek, T. Apih, and M. U. Mikac, Phys. Rev. B 55, 12161 (1997).

[11] N. Nakayama, N. Nakamura, and H. Chihara, Bull. Chem. Soc. Japan 60, 99 (1987). tion stands out with a very low value of $\lambda(1.25)$ in comparison with the comparable values (within experimental errors) for all other compounds at about 3.0. Another important parameter that can be estimated and compared is the rms diffusion length $z_{\mathrm{av}}=\left(2 D_{\mathrm{eff}} t\right)^{1 / 2}$, where $t$ represents the time duration of the experiment [10]. Estimated values of this length are presented in Table 1.

Based on these results a general conclusion could be drawn that the substitution of an impurity slows down the collective dynamics of the aggregates due to increased pinning, even though the pre-transitional behaviour does not seem to vary that much. The case of TMA substitution is different : the dynamics slows down considerably and the observed critical dynamics is perceptibly insensitive to the critical behaviour of the order parameter concerned.

\section{Acknowledgements}

The work reported here is in part supported by the office of Naval Research (Grant No. N00014-97-1-0994) under the Indo-US joint research project.

[12] R. K. Subramanian, K. Venu, and V. S. S. Sastry, J. Phys.; Condensed Matter Physics 6, 137 (1994); 6, 2377 (1994); R. K. Subramanian, S. Uma Maheswari, K. Venu, and V. S. S. Sastry, Z. Naturforsch. 51 a, 745 (1996).

[13] S. Uma Maheswari, K. Venu, and V. S. S. Sastry, submitted for publication 1999.

[14] A. M. Fajdiga, T. Apih, J. Dolinsek, R. Blinc, A. P. Levanyuk, S. A. Minyukov, and D. C. Ailion, Phys. Rev. Lett. 69, 2721 (1992).

[15] J. Dolinsek, A. M. Fajdiga-Bulat, T. Apih, R. Blinc, and D. C. Ailion, Phys. Rev. B 50, 9729 (1994).

[16] E. O. Stejskal and J. E. Tanner, J. Chem. Phys. 42, 288 (1965).

[17] S. Uma Maheswari, K. Venu, and V. S. S. Sastry, J. Phys.: Condensed Matter Physics 11, 5065 (1999).

[18] S. Uma Maheswari, K. Venu, and V. S. S. Sastry, submitted for publication 1999 .

[19] J. Dolinsek and R. Blinc, J. Phys.: Condensed Matter Physics 21, 705 (1988); F. Milia, G. Papavassiliou and E. Ginnakopoulos, Z. Naturforsch. 45 a, 323 (1989).

[20] R. Blinc, D. C. Ailion, P. Prelovsek, and V. Rutar, Phys. Rev. Lett. 50, 67 (1983). 